\title{
Cell culture-based production of defective interfering particles for influenza antiviral therapy
}

\author{
Milena A. Wasik ${ }^{1}$ - Luca Eichwald ${ }^{1} \cdot$ Yvonne Genzel $^{1} \cdot$ Udo Reichl $^{1,2}$
}

Received: 4 October 2017 / Revised: 14 November 2017 / Accepted: 15 November 2017 / Published online: 5 December 2017

(C) The Author(s) 2017. This article is an open access publication

\begin{abstract}
Defective interfering particles (DIPs) lack an essential portion of the virus genome, but retain signals for replication and packaging, and therefore, interfere with standard virus (STV) replication. Due to this property, DIPs can be potential antivirals. The influenza A virus DIP DI244, generated during propagation in chicken eggs, has been previously described as a potential candidate for influenza antiviral therapy. As a cell culture-based manufacturing process would be more suitable to fulfill largescale production needs of an antiviral and enables full process control in closed systems, we investigated options to produce DI244 in the avian cell line AGE1.CR.pIX in chemically defined suspension culture. With a DI244 fraction of 55.8\% compared to STV, the highest DI244 yield obtained from 50 million cells was $4.6 \times 10^{9} \mathrm{vRNA}$ copies $/ \mathrm{mL}$ at $12 \mathrm{~h}$ post infection. However, other defective genomes were also detected. Since these additionally produced defective particles are non-infectious, they might be still useful in antiviral therapies. In case they would interfere with quality of the final product, we examined the impact of virus seeds and selected process parameters on DI244 yield and contamination level with other defective particles. With a DI244 fraction of $5.5 \%$, the yield obtained was $1.7 \times 10^{8} \mathrm{vRNA}$ copies $/ \mathrm{mL}$ but now without additional defective genomes. Although the DI244 yield might be decreased in this case, such controlled manufacturing conditions are not available in chicken eggs. Overall, the application of these findings can support design and optimization of a cell culture-based production process for DIPs to be used as antivirals.
\end{abstract}

Keywords Defective interfering particles $\cdot$ DI244 antiviral $\cdot$ Influenza A virus $\cdot$ Cell culture $\cdot$ Suspension cell line

\section{Introduction}

Influenza viruses are enveloped viruses of the Orthomyxoviridae family with a segmented genome that consists of eight negativestranded RNA segments. Influenza viruses can cause respiratory illness known as flu. Annual flu epidemics worldwide are estimated by the WHO to result in about 3 to 5 million cases of severe illness, and about 250,000 to 500,000 deaths. The most effective way to prevent flu is vaccination, but influenza vaccines do not always match the strains that are currently circulating. Two classes of antiviral drugs against influenza are currently

Milena A. Wasik

wasik@mpi-magdeburg.mpg.de

1 Bioprocess Engineering, Max Planck Institute for Dynamics of Complex Technical Systems, Sandtorstrasse 1,

39106 Magdeburg, Germany

2 Bioprocess Engineering, Otto von Guericke University Magdeburg, Universitaetsplatz 2, 39106 Magdeburg, Germany available, to which however, virus resistance has become more and more frequent (Mc Mahon and Martin Loeches 2017, WHO 2017]. This raises the need for new antiviral drugs.

The use of defective interfering particles (DIPs), which reduce infectious load and stimulate the adaptive and innate immunity (Scott et al. 2011a), is one such new approach for an influenza antiviral therapy. One candidate is DI244, described by Dimmock et al. (Dimmock et al. 2008), which differs from infectious influenza A virus only by a single internal deletion in the largest genomic segment 1 that codes for the polymerase basic protein 2 (PB2). The DI244 RNA comprises $395 \mathrm{nt}$ instead of $2341 \mathrm{nt}$, but contains the termini of the RNA that carry the replication and packing signals essential for the DIPs to be propagated and packaged. Due to the defective virus genome, DI244 and other DIPs are not able to replicate on their own, but require the presence of a completely functional standard virus (STV), which provides the missing protein(s). DIPs interfere with the replication of STV in co-infected cells, and thus, can efficiently reduce the infectious particle production of some influenza strains (Frensing et al. 2014). In 
particular, it was shown that DI244 protects mice and ferrets from lethal infection caused by a number of different influenza A viruses, an influenza B virus strain, and a murine pneumovirus strain, suggesting further development as a broad-acting antiviral (Dimmock et al. 2012, Scott et al. 2011b, Easton et al. 2011).

Until now, DI244 has been grown in embryonated chicken eggs (Dimmock et al. 2008). While egg-based influenza vaccine production is well understood and produces consistent results, it has several disadvantages, i.e., it is poorly scalable, involves the possibility of bacterial contamination that necessitates the addition of antibiotics, and some people suffer from allergic reactions to egg components that may be present in the product (Minor et al. 2009, Perdue et al. 2011, Chung 2013). Influenza virus propagation in cell culture overcomes these problems (WHO 1995). Moreover, cell culture-based production can be initiated without long lead times and takes place in a completely closed and aseptic environment with full control of cultivation conditions, substrates, and quality. Furthermore, it was shown for one influenza vaccine that it contained substantial amounts of defective RNA that arose during production in chicken eggs (Gould et al. 2017). This can be true for other influenza vaccines as well. Whether vaccine production without accumulation of defective RNAs is possible in cell culture has not been reported yet.

In this study, we investigated the replication of DI244 in a designer cell line established to replace primary chicken cells in vaccine production (Jordan et al. 2009). The cell line AGE1.CR.pIX originates from the Muscovy duck, is well characterized, and has been reported to support fast propagation of influenza viruses (Lohr et al. 2009). Suspension growth of this cell line in a chemically defined medium allows the development of easily scalable processes, fulfilling all requirements in state-of-the-art vaccine manufacturing. Usually, the formation of DIPs is unwanted in influenza vaccine manufacturing, since DIPs can lower the virus yields (Frensing et al. 2014). Therefore, low multiplicity of infection (MOI) conditions are typically chosen for virus propagation. Little is known however, which conditions are required for cell culture-derived production of high-yield DIP preparations for animal and clinical studies, and for establishment of commercial manufacturing processes.

In this study, we characterized specific properties of a virus seed used for DI244 production in AGE1.CR.pIX cells and the impact of cultivation conditions on DI244 yield and contamination level with additional defective genomes, i.e., the impact of MOI and trypsin activity. In addition, we have established a RT-qPCR assay which enables the quantification of DI244 and STV copy numbers based on segment 1 , which cannot be monitored by other titration methods, i.e., $\mathrm{TCID}_{50}$ and PFU assay (infectious virus particles) or HA assay (total number of virus particles). Based on small-scale experiments, we propose a cell culture-based process for DIPs generation, specifically DI244, for manufacturing of this new class of antivirals.

\section{Materials and methods}

\section{Cells}

The cell line AGE1.CR.pIX was provided by ProBioGen $\mathrm{AG}$ and cultivated in $125 \mathrm{~mL}$ vented and baffled shaking flasks (wv, working volume $50 \mathrm{~mL}$ ) at $185 \mathrm{rpm}, 37^{\circ} \mathrm{C}$, and $5 \% \mathrm{CO}_{2}$. The chemically defined CD-U3 medium (Biochrom/Merck) was supplemented with $2 \mathrm{mM}$ glutamine (Sigma), $2 \mathrm{mM}$ alanine (Sigma), and $10 \mathrm{ng} / \mathrm{mL}$ recombinant insulin-like growth factor $\left(\mathrm{LONG}^{3} \mathrm{IGF}\right.$, Sigma). Cells were passaged twice a week by dilution to the starting cell concentration of $8 \times 10^{5}$ cells $/ \mathrm{mL}$ using fresh medium.

Adherent MDCK cells (ECACC, \#84121903), used for the innocuity test (see Results, UV irradiation for inactivation of standard virus) and $\mathrm{TCID}_{50}$ assay (see Measurement of virus titers), were maintained in T75 flasks containing $50 \mathrm{~mL}$ GMEM (GIBCO) supplemented with $10 \%(v / v)$ fetal calf serum (PAN Biotech) and $1 \%(v / v)$ peptone $(\mathrm{Lab} \mathrm{M})$ at $37^{\circ} \mathrm{C}$ in a $5 \% \mathrm{CO}_{2}$ atmosphere.

\section{Viruses}

The egg-derived DI244/PR8 virus, previously described (Dimmock et al. 2008) and here referred to as DI244/ STV, was provided by Nigel J. Dimmock from the University of Warwick (UK). For adaptation to AGE1.CR.pIX cells and to generate virus seed for subsequent experiments, DI244/STV was serially passaged using a trypsin activity of $3 \times 10^{-6} \mathrm{U} /$ cell. The first passage was conducted by directly infecting cells with $50 \mu \mathrm{L}$ of DI244/STV. The resulting virus showed a TCID $_{50}$ titer of $7.2 \times 10^{4}$ infectious virions $/ \mathrm{mL}$ and a HA titer of $1.37 \mathrm{log}$ $\mathrm{HAU} / 100 \mu \mathrm{L}$ at $12 \mathrm{hpi}$. For the second passage, MOI $10^{-3}$ (based on $\mathrm{TCID}_{50}$ assay) was used and the virus was harvested at $24 \mathrm{hpi}$. The third passage was performed at MOI $10^{-6}$ and resulted in the DI244 working virus seed, which showed a $\mathrm{TCID}_{50}$ titer of $5.5 \times 10^{5}$ infectious virions $/ \mathrm{mL}$ and a HA titer of $1.45 \log \mathrm{HAU} / 100 \mu \mathrm{L}$ at $24 \mathrm{hpi}$. The fourth passage was conducted at MOI $10^{-8}$ and resulted in the DIP-free STV working virus seed, which showed a $\mathrm{TCID}_{50}$ titer of $2.1 \times 10^{6}$ infectious virions $/ \mathrm{mL}$, a HA titer of $1.82 \log \mathrm{HAU} / 100 \mu \mathrm{L}$, and $6.8 \times 10^{9} \mathrm{STV}$ vRNA 
copies/mL by RT-qPCR at 48 hpi. No defective genomes including DI244 were detected by RT-PCR. In addition, a STV-free DI244 seed was produced by UV irradiation (see below) of the DI244 working virus seed.

\section{UV irradiation}

UV irradiation to destroy the full-length segments of STV (Dimmock et al. 2008) for co-infection experiments (see Results, UV irradiation for inactivation of standard virus) was performed in 12-well plates with a surface area of $4 \mathrm{~cm}^{2}$. A volume of $350 \mu \mathrm{L}$ per well (liquid layer of approx. $1 \mathrm{~mm}$ ) of the DI244 working virus seed was exposed for $8.5 \mathrm{~min}$ to UV light $(254 \mathrm{~nm})$ in the PCR Workstation Pro (VWR Peqlab) with a distance of about $65 \mathrm{~cm}$ to the two UV tubes (each $25 \mathrm{~W}$ ).

\section{Infections}

After inoculating $8 \times 10^{5}$ AGE1.CR.pIX cells $/ \mathrm{mL}$, the cells were grown 3-4 days in shaker flasks. Cells were diluted without any washing steps to a cell concentration of $1 \times 10^{6}$ cells $/ \mathrm{mL}$ in a volume of $50 \mathrm{~mL}$ using fresh medium. Calculated volumes of virus seed for the desired MOIs between $10^{-1}$ and $10^{-8}$ (as indicated for each experiment), based on the $\mathrm{TCID}_{50}$ assay (see Measurement of virus titers), and trypsin stock solution (500 units/mL, GIBCO) for the desired trypsin activity (as indicated for each experiment) were added directly to the cell suspension. Virus samples were collected at $36 \mathrm{~h}$ post infection (hpi) if not indicated otherwise. Samples were centrifuged at $300 \mathrm{~g}$ for $5 \mathrm{~min}$ to remove cells and supernatant was stored at $-80{ }^{\circ} \mathrm{C}$ until RNA isolation.

For the innocuity test, confluent cultures of adherent MDCK cells were washed twice with PBS. $1 \mathrm{~mL}$ of UVirradiated DI244 working virus seed was added to the cells in $50 \mathrm{~mL}$ serum-free GMEM (GIBCO) containing 1\% $(v / v)$ peptone (Lab M) and $5 \mathrm{U} / \mathrm{mL}$ trypsin (GIBCO, \#27250-018). Virus samples were collected at $72 \mathrm{hpi}$. Samples were centrifuged at $300 \mathrm{~g}$ for $5 \mathrm{~min}$ and the supernatant was stored at $80{ }^{\circ} \mathrm{C}$ until HA assay.

\section{Measurement of virus titers}

Infectious virus particle concentrations were measured using a tissue culture infectious dose $50\left(\mathrm{TCID}_{50}\right)$ assay by titration of supernatant samples on MDCK monolayers as described previously (Genzel and Reichl 2007). Titers were expressed as infectious virions $/ \mathrm{mL}$.

Total virus particle concentrations were determined using a hemagglutination assay as described previously (Kalbfuss et al. 2008). Titers are expressed as $\log _{10}$ HA units per test volume $(\log \mathrm{HAU} / 100 \mu \mathrm{L})$.

\section{Segment-specific RT-PCR for detection of defective genomes}

RNA was isolated from $150 \mu \mathrm{L}$ supernatant using the NucleoSpin RNA Virus Kit (Macherey-Nagel) according to the manufacturer's instructions.

The reverse transcription (RT) of isolated RNA to cDNA as well as the amplification of all eight influenza virus genome segments with segment-specific primers for detection of defective genomes was performed as described previously (Frensing et al. 2014). The RT-PCR products were directly analyzed on a $1 \%$ agarose gel using electrophoresis.

\section{Segment-specific RT-qPCR for DI244 and STV quantification}

To determine viral RNA (vRNA) copy numbers, a reverse transcription quantitative PCR (RT-qPCR) was performed using RNA reference standards for the targeted RNA as described previously (Frensing et al. 2014). Briefly, pUC19 plasmids with inserted full-length segment 1 (FL1) or DI244 cDNA were cloned, respectively. Plasmids were subjected to PCR with the primer pair Seg-1-Uni-for (AGCGAAAG CAGGTCAATTAT) and Seg-1-Uni-T7-rev (TAATACGA CTCACTATAGGGAGTAGAAACAAGGTCGTTTT TAAAC) to introduce a $\mathrm{T} 7$ promoter sequence in the desired orientation into the PCR product. PCR products were in vitro transcribed to vRNA. Absolute vRNA copy numbers were determined as described previously (Frensing et al. 2014). In brief, a 10-fold dilution series of the corresponding vRNA reference standards and the undiluted isolated RNA were reverse transcribed with the polarity-specific and tagged primer Seg-1-tagRT-for (ATTTAGGTGACACTATAGAAGC GAGCGAAAGCAGGTCAATTATATTC). To distinguish DI244 from FL1, primer sets were used in qPCR, with one primer binding to the junction region of the defective segment, DI244-realtime-rev (GGAATCCCCTCAGTCTTC), and to the region of FL1, which is deleted from DI244, respectively, FL1-realtime-rev (CATTTCATCCTAAGTGCTGG), and one primer binding to the tag, which was prior to that introduced in reverse transcription, vRNA-tagRealtime-for (ATTTAGGT GACACTATAGAAGCG). The Rotor-Gene SYBR Green PCR Kit (Qiagen) and the Rotor-Gene Q real-time PCR cycler (Qiagen) were used for quantification following the manufacturer's recommendations. Viral RNA copy numbers in the samples (isolated RNA; see above) were calculated based on the vRNA reference standards with linear regression. Since the qPCR primers bind to extracellular vRNA and theoretically only one copy of vRNA is packed per virion, the qPCR data is equivalent to the total number of virus particles. Standard curves showed strong linear correlations $(>0.99)$ over eight orders of magnitude for DI244 and seven orders of magnitude for FL1, respectively (data not shown). Amplification 
efficiency was between 98 and 108\%. The lowest concentration at which linearity was retained in the standard curve was $2.2 \times 10^{3}$ DI244 copies $/ \mu \mathrm{L}$ and $3.8 \times 10^{3} \mathrm{FL} 1$ copies $/ \mu \mathrm{L}$, respectively. The inter-assay coefficient of variation for the copy numbers was $6.4 \%$ for DI244 vRNA and $5.1 \%$ for FL1 vRNA, respectively.

\section{Results}

\section{Generation of a defined DI244 working virus seed for cell culture experiments}

Influenza virus propagation in embryonated chicken eggs and animal cells can favor the generation of various defective genomes. Indeed, besides DI244, we detected multiple uncharacterized defective genomes in segments 1, 2, 3, 4, and 6 in the DI244/STV material provided (Fig. 1a). To generate a better defined working virus seed for cell culture experiments, we investigated if these additional defective genomes in DI244/STV can be depleted after several replication rounds in cell culture. Initially, we chose the Madin-Darby Canine Kidney (MDCK) cell line MDCK.SUS2, adapted to serum-free suspension growth, as a substrate, since MDCK cells support the replication of influenza virus to high titers and are already licensed as host cells for influenza vaccine manufacturing (Peschel et al. 2013, Hegde 2015). However, even after several passages of DI244/STV in MDCK.SUS2 cells, additional defective genomes of segments 1, 2, 3, 4, and 6 were still detected by RT-PCR (data not shown). Since the generation of defective particles is cell dependent (De and Nayak 1980, Crumpton et al. 1981), we tested different cell lines, e.g., the cell line AGE1.CR.pIX, which was specifically designed for vaccine manufacturing in chemically defined media (Jordan et al. 2009). Moreover, the AGE1.CR.pIX suspension cell line was shown to propagate influenza virus efficiently (Lohr et al. 2012). We infected AGE1.CR.pIX cells with 10-fold dilutions of DI244/STV. In the virus material harvested after one passage in AGE1.CR.pIX cells, no defective genomes of segments 4 and 6 were detected by RT-PCR (Fig. 1b). By subjecting this material to two more passages in AGE1.CR.pIX cells, we obtained a DI244 working virus seed without noticeable defective genomes besides DI244 (Fig. 1c). Since this RT-PCR can only serve as a visual quality control, the newly established RT-qPCR was used to determine DI244 vRNA copy numbers in the samples.

Assuming an egg volume of $10 \mathrm{~mL}$ (allantoic fluid), the egg-derived virus material showed the highest DI244 vRNA concentration with $20.5 \times 10^{9}$ copies $/ \mathrm{mL}$ (Fig. 1d). The virus harvested after one passage from $50 \times 10^{6}$ AGE1.CR.pIX cells showed a DI244 yield of $4.6 \times 10^{9}$ copies $/ \mathrm{mL}$, which decreased by about one order of magnitude after three passages to $1.7 \times 10^{8}$ copies $/ \mathrm{mL}$ for the resulting DI244 working virus seed (Fig. 1d). The percentage of DI244 differed significantly for all DI244 containing virus materials. It started with $37.0 \%$ in the egg-derived material, increased to $55.8 \%$ after one passage in cell culture, but decreased to $5.5 \%$ for the working virus seed free of additional defective genomes (Fig. 1e).

\section{Screening for optimal cultivation conditions for DI244}

For a systematic characterization of the impact of selected process conditions on DI244 yield and on the generation of additional defective genomes, we varied both MOI and trypsin activity, which are critical parameters in influenza vaccine production (Genzel and Reichl 2009). For all experiments, the DI244 working virus seed (Fig. 1c) was used. Since defective genomes are more likely to occur at high MOIs (Heldt et al. 2013), we started with a low MOI $\left(10^{-5}\right)$ to reduce the risk of de novo generation of additional defective genomes and increased MOI stepwise. The addition of trypsin at time of infection is required for hemagglutinin cleavage to facilitate virus infection. A trypsin activity of $10^{-6} \mathrm{U} /$ cell was previously shown to be the most effective in influenza virus production in AGE1.CR.pIX cells (Lohr et al. 2012). Therefore, in addition to MOI, trypsin activities in the range $10^{-5}$ to $10^{-7} \mathrm{U} /$ cell were also tested. Furthermore, supernatants from four different harvesting time points were characterized by RT-PCR. The harvests were analyzed for DI244 and possible contamination with other defective genomes in segments 1,2, and 3, since defective RNAs are more likely to be generated from polymerase coding segments (Nayak et al. 1985).

We were able to observe three trends after infection of AGE1.CR.pIX cells with the DI244 working virus seed at trypsin activities in the range $10^{-5}$ to $10^{-6} \mathrm{U} /$ cell: A variety of additional defective genomes in segments 2 and 3 were generated at MOI $10^{-5}$ (Fig. 2a). No additional defective genomes were generated at MOI $10^{-6}$ and $10^{-7}$ (Fig. 2b). Infections at MOI $10^{-8}$ resulted in the loss of DI244 (Fig. 2c). No virus replication was observed for all MOIs at a trypsin activity of $10^{-7} \mathrm{U} /$ cell (Fig. 2d). Obviously, this trypsin activity was too low for successful virus uptake and initiation of virus replication. We screened further under conditions shown in Fig. 2b and measured the DI244 copy number by RT-qPCR. Interestingly, the DI244 copy numbers were stable, corresponding to the initial DI244 copy number of the DI244 working virus seed $\left(1.7 \times 10^{8}\right.$ copies $\left./ \mathrm{mL}\right)$. The highest copy number was achieved $12 \mathrm{hpi}$ with only a slight decrease over the next $36 \mathrm{~h}$ (Fig. 2e). Overall, this data suggests that the MOIs and trypsin activities should be adjusted to the range $10^{-6}$ to $10^{-7}$ and $10^{-5}$ to $10^{-6}$, respectively, for cell culturederived DI244 production. 
a

b

C
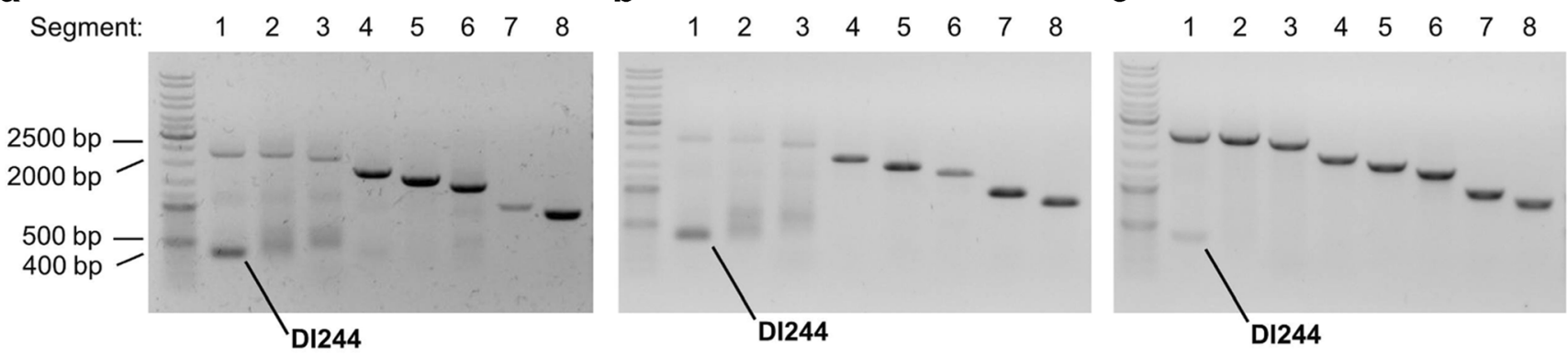

d

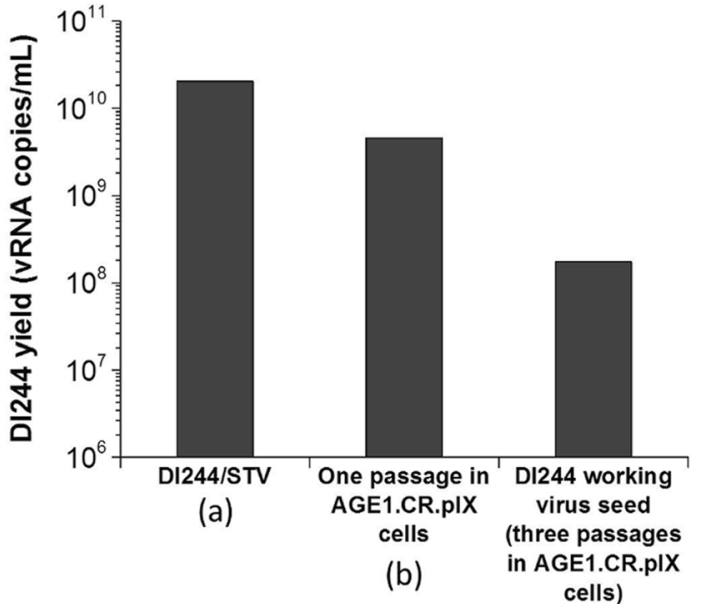

(c) e

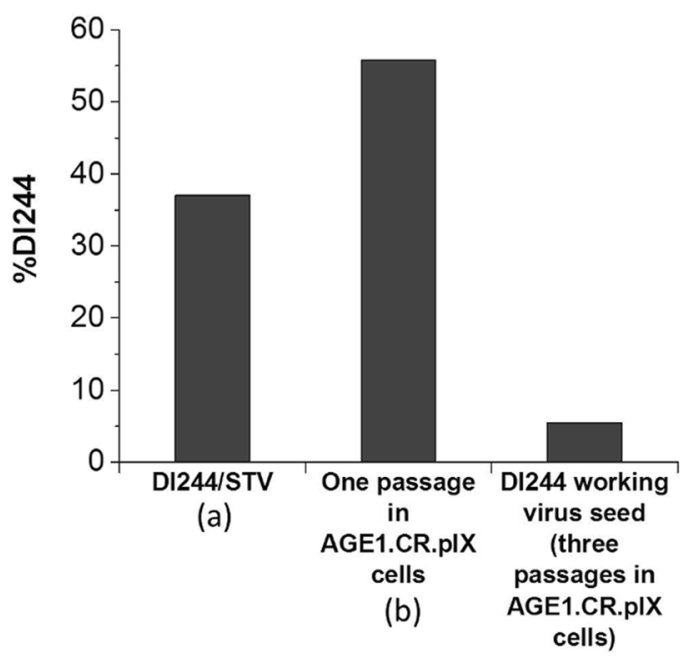

(c)
Fig. 1 Gel electrophoretic analysis of segment-specific RT-PCR to detect defective influenza virus genomic RNAs (all eight influenza genome segments were amplified) and quantification of DI244 by RT-qPCR. a RT-PCR of DI244/STV. b RT-PCR of DI244/STV after one passage in AGE1.CR.pIX cells; AGE1.CR.pIX cells were infected with $50 \mu \mathrm{L}$ DI244/STV and supernatant was harvested at 12 hpi. c RT-PCR of DI244 working virus seed after three passages in AGE1.CR.pIX cells.

\section{UV irradiation for inactivation of standard virus}

After UV irradiation, infectivity of STV was measured by $\mathrm{TCID}_{50}$ assay for a range of UV exposure times $(10 \mathrm{~s}$ to $8.5 \mathrm{~min})$. The specific inactivation rate of this first order reaction was $3.1 \mathrm{~min}^{-1}$ (linear range 0.33 to $2.0 \mathrm{~min}$ ). Complete loss of infectivity $\left(\mathrm{TCID}_{50}\right.$ titer below the detection limit) was observed for UV exposure times of $4.5 \mathrm{~min}$ and above (Fig. 3a). Further RT-PCR analysis showed that FL1 was not amplified after $4.5 \mathrm{~min}$ of UV irradiation (Fig. 3b), indicating degradation of FL1. To ensure that no replication competent virus was present after UV irradiation, the innocuity of STV was tested for two serial passages in MDCK cells by monitoring HA titers. The necessary UV exposure time to ensure complete inactivation of STV was identified as $8.5 \mathrm{~min}$ (data not shown). For this time point, RT-PCR showed that all larger segments (2341 to $1778 \mathrm{nt}$ ) FL1, 2, 3, and 4 were destroyed (Fig. 3c), confirming again complete inactivation of STV. In
Supernatant was harvested at 24 hpi. d Copy numbers of DI244 vRNA in virus material obtained from one egg $(10 \mathrm{~mL}$ assumed for estimation of yield) or $50 \times 10^{6}$ AGE1.CR.pIX cells in $50 \mathrm{~mL}$ working volume (corresponding to samples from Fig. $1 \mathrm{a}, \mathrm{b}$, and c). Measurements were performed in triplicates (error too small to be shown). e Percentage of DI244 in virus material shown in Fig. $1 \mathrm{~d}$, measured by RT-qPCR (error too small to be shown)

addition, it was tested, whether DI244 replication was affected by UV irradiation for $8.5 \mathrm{~min}$. Therefore, AGE1.CR.pIX cells were co-infected with DIP-free STV working virus seed (MOI $10^{-4}$, i.e., $1.4 \times 10^{5} \mathrm{STV}$ vRNA copies $/ \mathrm{mL}$ ) and the same concentration of UV irradiated $(8.5 \mathrm{~min})$ DI244. At $36 \mathrm{hpi}$, an increase in vRNA copy numbers was observed for STV and DI244 by RT-qPCR (Fig. 3d). Hence, DI244 RNA was not significantly affected by UV irradiation due to its smaller genome size compared to the full-length infectious viral genome. This finding corresponds to the RT-PCR gel that shows a band for DI244 even after 8.5 min of UV irradiation (Fig. 3c).

\section{Impact of initial DI244/STV ratio on DI244 yield}

By analyzing the effect of MOI on DI244 propagation, we found that MOIs in the range $10^{-6}$ to $10^{-7}$ showed the most promising results as the generation of additional defective 

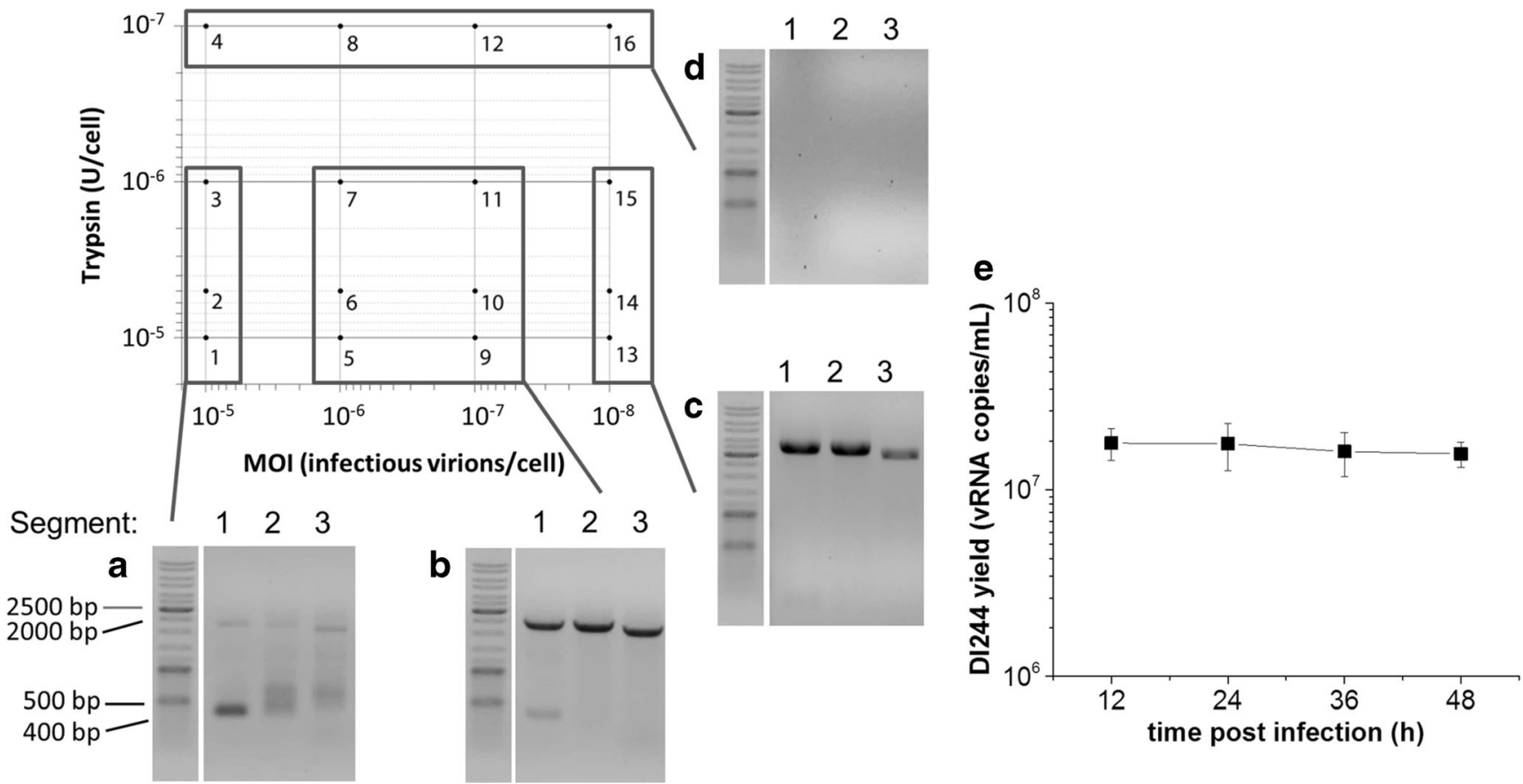

Fig. 2 Trends observed during infection of AGE1.CR.pIX cells with the DI244 working virus seed for different MOIs and trypsin activities. a (\#3) Infections at MOI $10^{-5}$ and trypsin activities in the range $10^{-5}$ and $10^{-6} \mathrm{U} /$ cell showed a strong band for DI244 as well as de novo generation of defective genomes in segments 2 and 3. b (\#7) Infections at the MOIs $10^{-6}$ and $10^{-7}$ and trypsin activities in the range $10^{-5}$ and $10^{-6} \mathrm{U} /$ cell did not favor the generation of additional defective genomes. c (\#15) Infections at MOI $10^{-8}$ and trypsin activities in the range $10^{-5}$ and

$10^{-6} \mathrm{U} /$ cell resulted in the loss of DI244. d (\#8) Infections with a trypsin activity of $10^{-7} \mathrm{U} /$ cell showed no viral RNA bands by RT-PCR for all MOIs. Samples, collected at $36 \mathrm{hpi}$, are shown. Numbers above the gel pictures represent the segment numbers; numbers associated with each gel represent the specific experimental condition. e RT-qPCR analysis of vRNA of DI244 () over time at an MOI of $10^{-5}$ and a trypsin activity of $10^{-6}$. Error bars indicate standard deviation of three independent biological experiments

genomes could be prevented (Fig. 2b). However, the fraction of DI244 was rather low with $<2 \%$. It has to be taken into account, however, that the infection was performed with the DI244 working virus seed which had already a low DI244 fraction of $5.5 \%$ (Fig. 1e). Most likely, this resulted in poor replication conditions of DI244 compared to STV. To determine the impact of the DI244/STV ratio of working seeds on DI244 yield in virus harvests, we infected cells with the same amount or an excess of DI244 over STV. For this experiment, we produced a DIP-free STV working virus seed using a MOI of $10^{-8}$ and a trypsin activity of $3 \times 10^{-6} \mathrm{U} /$ cell, respectively, and a STV-free DI244 stock by UV irradiation. Then, we infected AGE1.CR.pIX cells at a MOI of $10^{-6}$ with STV and added DI244 in the vRNA copy number ratios 1:1, 1.5:1, 2:1, 10:1, and 1:10, determined by RT-qPCR. Higher DI244/STV ratios were not tested, since this could lead to interference of DI244 with STV, and thus, inhibition of DIP replication, resulting in reduced yields (Scott et al. 2011a, Akkina et al. 1984, Stauffer Thompson et al. 2009). For harvests at 36 hpi and all ratios tested, DI244 yields were in the same order of magnitude at about 2.5 to $10.4 \times 10^{7}$ vRNA copies $/ \mathrm{mL}$ (Fig. 4a) with a DI244 fraction not exceeding $1.1 \%$ (Fig. 4b). No additional defective genomes were detected by

RT-PCR (Fig. 4c). This is according to the results shown in Fig. 2b, where we showed that a low MOI is preventing the generation of additional defective genomes, but does not benefit DI244 production.

\section{Impact of MOI for infections with same DI244/STV ratio}

To better characterize DI244 replication in cell culture and evaluate options to increase the DI244 content, we quantitatively analyzed the MOI dependency on DI244 yield. Using the virus seeds generated for the experiments above, we infected AGE1.CR.pIX cells with a constant DI244/STV ratio of 1:1, increased the MOI stepwise, and measured the DI244 vRNA concentration by RT-qPCR. A constant DI244/STV ratio of 1:1 was chosen to give both DI244 and STV the same chance of replication. For $36 \mathrm{hpi}$, we observed that the DI244 yield as well as the DI244 fraction increased proportionally to MOI (Fig. 5a, b). However, at MOIs $10^{-5}$ to $10^{-2}$, the STV yield was higher. A maximum of $1.8 \times 10^{9}$ DI244 vRNA copies $/ \mathrm{mL}$ was obtained for MOI $10^{-1}$, where the DI244 showed a slight replication advantage over STV (Fig. 5a). Overall, we were able to increase the DI244 yield by two 
a

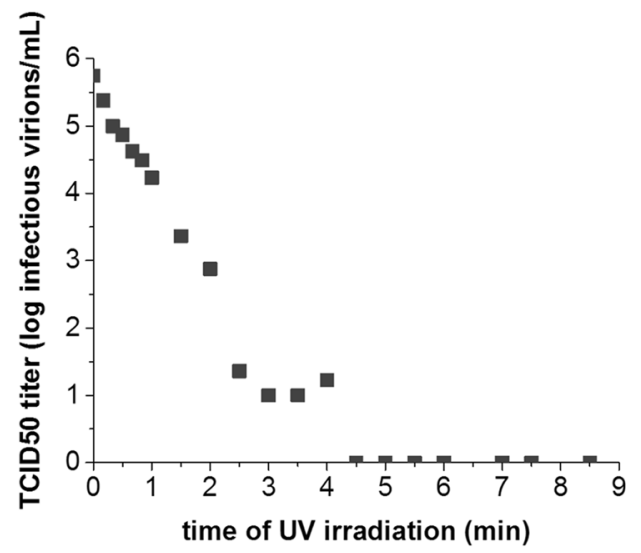

C

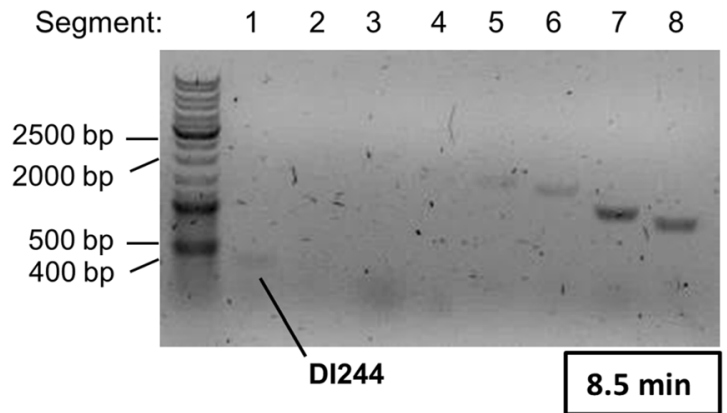

b
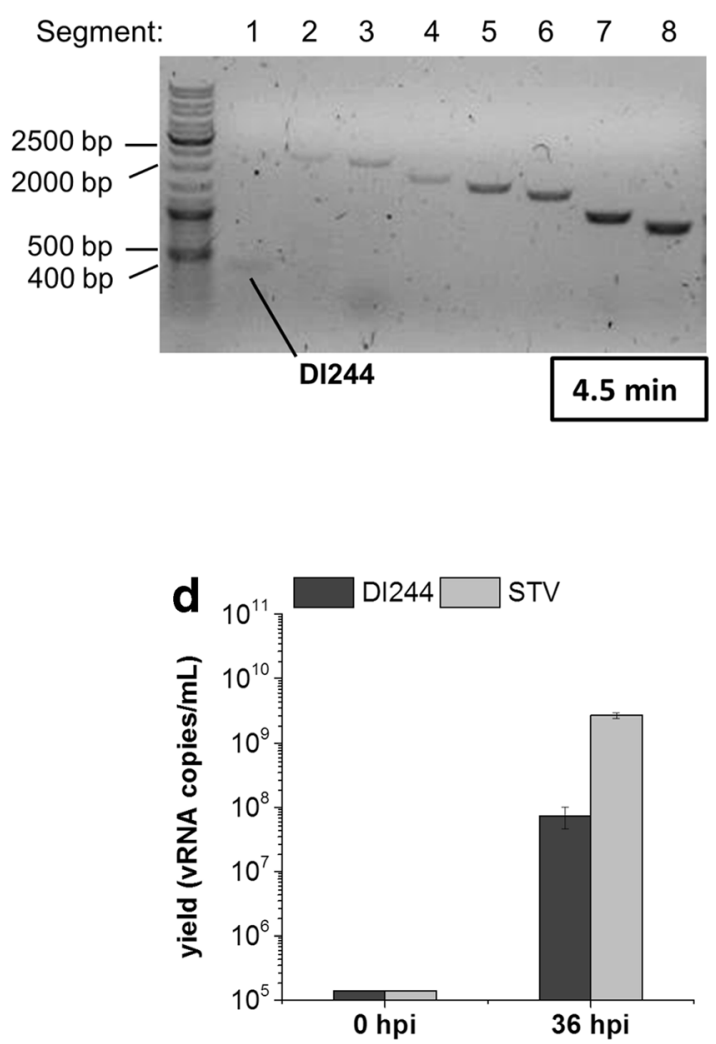

Fig. 3 Inactivation of DI244 working virus seed by UV irradiation. a Decrease in $\mathrm{TCID}_{50}$ with UV irradiation time. Samples, UV irradiated for $4.5 \mathrm{~min}$ or $8.5 \mathrm{~min}$, respectively, were measured in independent triplicates (error too small to be shown). b Gel electrophoretic analysis of segment-specific RT-PCR of all eight influenza genomic RNAs (one to eight) and DI244 RNA in the DI244 working virus seed after UV irradiation for $4.5 \mathrm{~min}$. $\mathbf{c}$ Gel electrophoretic analysis of segment-specific RT-
PCR of all eight influenza genomic RNAs (one to eight) and DI244 RNA in the DI244 working virus seed after UV irradiation for $8.5 \mathrm{~min}$. d DI244 activity in AGE1.CR.pIX cells after UV irradiation for $8.5 \mathrm{~min}$ and subsequent infection with STV (MOI $10^{-4}$, ration 1:1). At $36 \mathrm{hpi}$, an increase in STV (light gray) as well as DI244 (dark gray) vRNA copy numbers was observed by RT-qPCR. Error bars indicate standard deviation of three independent biological experiments orders of magnitude and the DI244 fraction to $54.5 \%$, respectively. However, at all tested MOIs, defective genomes were generated in segments 1 (at about $1500 \mathrm{bp}$ ), 2, and 3 in addition to DI244 (Fig. 5c).

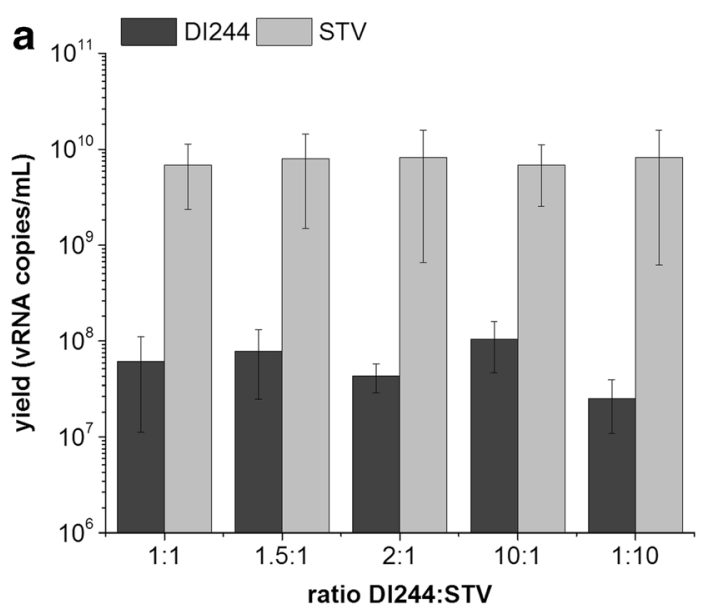

Fig. 4 a DI244 (dark gray) and STV (light gray) content and b percentage of DI244 after infection of AGE1.CR.pIX cells with the ratios 1:1, 1.5:1, $2: 1,10: 1,1: 10$ DI244/STV at MOI $10^{-6}$ using a trypsin activity of $10^{-6} \mathrm{U} /$

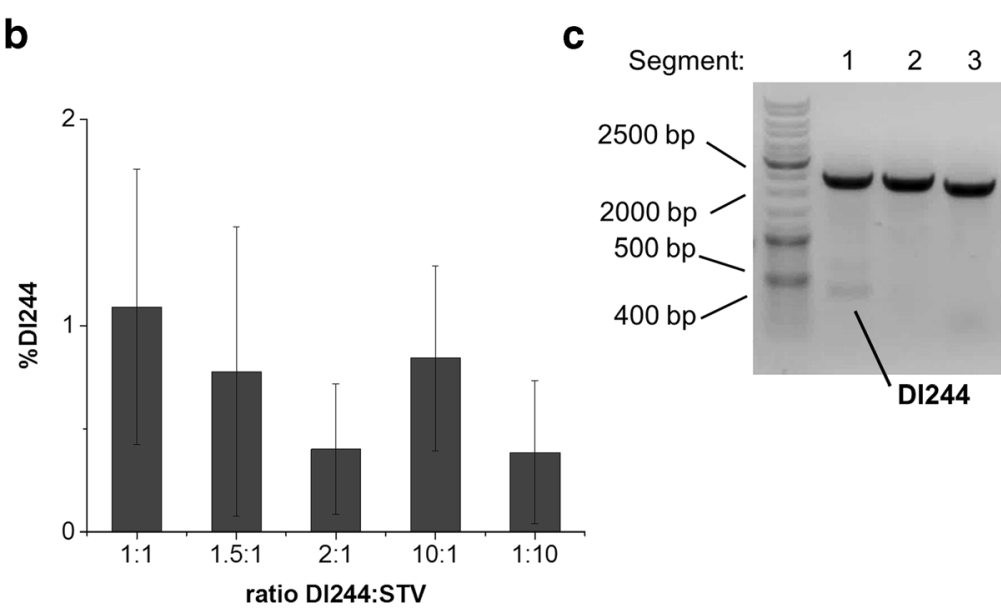

cell. Samples were collected at 36 hpi. c Gel picture of RT-PCR analysis representative for all tested ratios. Error bars indicate standard deviation of three independent biological experiments 


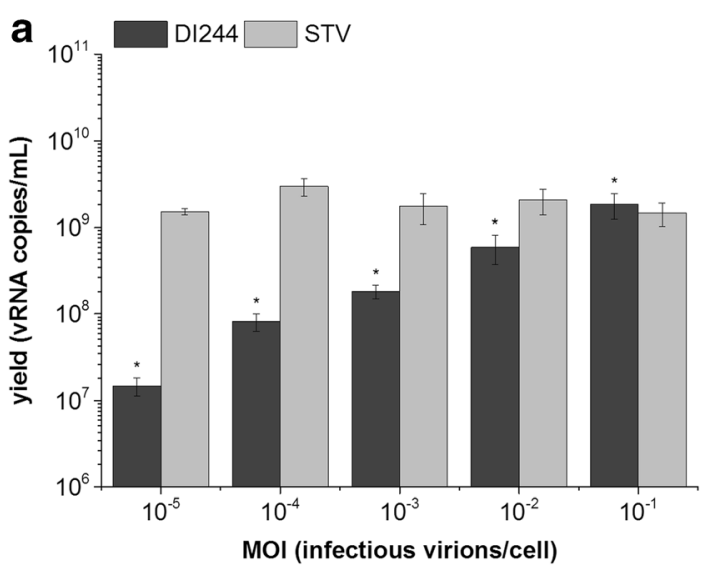

Fig. 5 a Yield and b percentage of DI244 (dark gray) and STV (light gray) after co-infection of AGE1.CR.pIX cells with ratio of 1:1 and different MOI using a trypsin activity of $10^{-6} \mathrm{U} /$ cell. Samples were collected at 36 hpi. c Gel picture of RT-PCR analysis representative for all tested

\section{Discussion}

\section{Cell culture-based DI244 production as an alternative to eggs}

Antiviral drug resistance of influenza viruses is an increasing problem and the demand for new options for antiviral therapy rises. DI244 was previously described to be a good candidate for blocking influenza virus infections (Dimmock et al. 2008). It was generated by a reverse genetic approach and passaged in embryonated chicken eggs to increase DI244 yields, which resulted in generation of numerous additional defective genomes. As an alternative, cell culture-based processes, which have been established over the last years, could be used for large-scale production in a completely contained and fully controlled environment. We therefore investigated options to generate suitable DI244 working virus seeds and established small-scale DI244 production in AGE1.CR.pIX suspension cells, which were specifically designed for vaccine manufacturing in chemically defined media.

\section{Comparison of DI244 yields in eggs and cell culture}

By replicating the egg-derived DI244/STV in AGE1.CR.pIX cells, we were able to eliminate defective genomes of segments 4 and 6. Also, the fraction of DI244 increased from $37.0 \%$ for the egg-derived to $55.8 \%$ for the cell culturederived harvests. Assuming an egg volume of $10 \mathrm{~mL}$ (allantoic fluid), the egg-derived virus material showed with $20.5 \times$ $10^{9}$ copies/mL a higher DI244 vRNA concentration then the virus harvested after one passage in AGE1.CR.pIX cells with $4.6 \times 10^{9}$ copies $/ \mathrm{mL}$. However, the cultivation was performed in small-scale shaking flasks with the relatively low concentration of $1 \times 10^{6}$ AGE1.CR.pIX cells $/ \mathrm{mL}$. This resulted in a

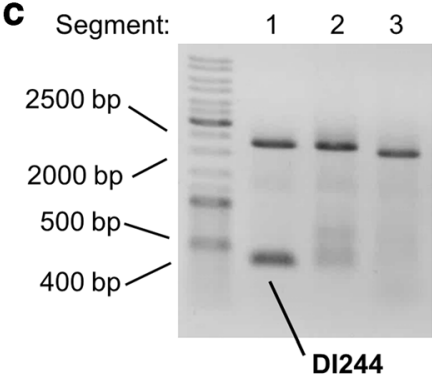

MOI. Error bars indicate standard deviation of three independent biological experiments $(* p<0.05$ for DI244 at all tested MOI by Kruskal-Wallis ANOVA) cell-specific yield of $4.6 \times 10^{3}$ DI244 vRNA copies/cell. In fully controlled bioreactors, however, AGE1.CR.pIX cells can be infected at concentrations up to $10 \times 10^{6}$ cells $/ \mathrm{mL}$ (Genzel et al. 2014). Thus, a $1 \mathrm{~L}$ bioreactor could yield $46 \times$ $10^{9}$ DI244 vRNA copies/mL within 1 or 2 days post infection. One clinical dose for mice has been reported to contain about $3.4 \times 10^{9}$ DI244 copies (Dimmock and Easton 2015). Thus, a $1 \mathrm{~L}$ bioreactor yield could be sufficient for providing 13.5 mouse doses per $\mathrm{mL}$ or a total of about 13,529 mouse doses. To gain the same yield, 244 fertilized eggs would be necessary, since an egg yields about six doses per $\mathrm{mL}$. Therefore, cell culture-based production is a valid alternative to eggbased manufacturing processes and could be even superior to egg-based production. However, whether our findings are 1:1 scalable needs further investigation. In addition, to further improve DI244 yields, high-cell-density cultures could be implemented. For example, in alternating tangential flow perfusion systems, up to $50 \times 10^{6}$ cells $/ \mathrm{mL}$ have been described for growth of AGE1.CR.pIX cells and subsequent influenza A virus production (Genzel et al. 2014). Also, other production cell lines should be tested, since the rates of influenza A virus replication as well as DIP generation vary greatly among different cell lines (De and Nayak 1980, Crumpton et al. 1981).

\section{Quantification of DI244 and dose input by RT-qPCR}

Although Dimmock and Easton specified DI244 copy numbers required for protection of mice, they used a specific DI244 virus protein mass for inoculation (Dimmock et al. 2008, Dimmock and Easton 2015). However, the estimation of DI244 input using protein concentration allows only a rough adjustment of the dose. Based on the established RT-qPCR assay, a more precise method for calculation of dose input for animal and human clinical 
trials is now available. Furthermore, this assay is also crucial for monitoring cell culture-based DI244 production and for establishment of mathematical models. Since virus titration assays are probably influenced by the presence of DIPs, and the TCID $_{50}$ titers of DI244 preparations are relatively low, the calculated volume for inoculation of a bioreactor with a specific MOI could be overestimated. Adjusting the virus input by DI244 copy numbers would be a more reliable method for DI244 virus seed characterization and for monitoring of production.

\section{Impact of selected process parameters and virus seeds on DI244 yield}

In a first step towards the design of DIP production processes, we examined the impact of the working virus seed as well as selected process parameters on DI244 yields. Among others, different ratios of DI244 to STV were tested with a constant MOI. To adjust these ratios, DI244 without other defective particles was used to reduce the risk of unspecific effects from additional defective genomes. Also, we chose a very low MOI of $10^{-6}$ to avoid the generation of defective particles other than DI244 (Fig. 2b). We observed that the initial ratio DI244/STV in the virus seed did not play a role in increasing the DI244 yield and fraction (Fig. 4a, b). However, at a MOI as low as $10^{-6}$, stochastic effects are likely, such as a low probability of co-infection of cells with DI244 and STV at first round(s) of replication. Furthermore, it was shown that up to $90 \%$ of influenza A virus-infected cells can fail to release infectious progeny at low MOI (Brooke et al. 2013). If such infections result mostly in non-productive progeny STV, DI244 cannot hijack (enough) FL1/PB2 for replication and preferential synthesis of DIPs as discussed previously (Stauffer Thompson et al. 2009, Dimmock and Easton 2015, Nayak et al. 1985). For this reason, we tested higher MOIs with a constant ratio of 1:1. We observed that the DI244 yield and fraction increased proportionally to MOI (Fig. 5a, b). The highest tested MOI of $10^{-1}$, where DI244 showed a slight replication advantage compared to STV, led to a sufficient DI244 yield comparable to the yield from one passage in AGE1.CR.pIX cells (Fig.1d, 5a). For large scale DI244 manufacturing, however, this would require the use of relatively large volumes of virus seeds and render production more complex or even impossible. Most likely, the use of MOIs in the range $10^{-4}$ to $10^{-2}$ would be feasible and further studies should be performed with other DI244/STV ratios to optimize infection conditions. However, at process conditions relevant for the manufacturing of DI244 antivirals, we also detected additional defective genomes in the harvests. As relatively high MOIs were required for obtaining reasonable yields, de novo generation of defective particles could not be avoided. This seems also true for egg-based antiviral production systems. Therefore, a compromise between contamination with other defective particles, yield, and virus seed volume used for initiation of infection has to be found. Whether the accumulation of such additional defective genomes is a disadvantage or not for the treatment of animals or humans cannot be decided at the moment. However, no adverse effects have been reported after giving recipients an influenza vaccine which contained substantial amounts of defective RNAs (Gould et al. 2017). Though the study suggests that defective RNAs do not present a health problem, it would be of considerable public interest to investigate the effect of defective RNAs in influenza vaccines. In case there is no negative impact on antiviral safety and efficacy, both cell culture and egg-based production systems could be used for industrial large-scale manufacturing.

\section{UV irradiation for inactivating STV in DI244 manufacturing}

For the use as an antiviral, infectious STV contaminating the produced DI244 harvests needs to be eliminated. Chemical inactivation is not possible, since it would also destroy DI244 RNA. Similar to egg-based DI244 production, UV irradiation of cell culture-derived DI244 could be an option to obtain a safe product, since it completely inactivates STV while leaving DI244 intact for intracellular replication. For the inactivation method used here, innocuity was demonstrated for STV containing samples after irradiation for $8.5 \mathrm{~min}$. However, regarding the establishment of large-scale production processes, it has to be taken into account that there is only very limited experience with UV irradiation for virus inactivation. Another promising option, not available in egg-based processes, would be the generation of cell lines, which stably express $\mathrm{PB} 2$, and thus, complement missing viral functions for DI244 production. The use of such a cell line would probably also minimize the risk of unwanted accumulation of other defective (interfering) particles and allow better designed studies to elucidate the mechanisms of DIP replication, not only in cell cultures, but also in animals and humans to explore the full potential of DIPs as antivirals. However, while such an approach would eliminate the need to use infectious STV for seed generation as well as avoid the establishment of inactivation processes, scale-up, and, in particular, optimization of cell-specific DIP yields could be issues to be addressed.

Acknowledgments Open access funding provided by Max Planck Society. We thank N. J. Dimmock and A. J. Easton from the University of Warwick for providing the DI244/PR8 egg-derived virus seed, T. Frensing for facilitating the cooperation to the University of Warwick, I. 
Jordan and V. Sandig from ProBioGen AG for providing the AGE1.CR.pIX cell line, P. Zmora and S. Fritzsche for critical comments on the manuscript, A.-M. Matthies, H. Rosenberg, and M. Gropper for scouting studies on DIP replication in animal cells, and I. Behrendt and N. Wynserski for technical assistance.

Compliance with ethical standards This article does not contain any studies with human participants or animals performed by any of the authors.

Conflict of interest The authors declare that they have no competing interests.

Open Access This article is distributed under the terms of the Creative Commons Attribution 4.0 International License (http:// creativecommons.org/licenses/by/4.0/), which permits unrestricted use, distribution, and reproduction in any medium, provided you give appropriate credit to the original author(s) and the source, provide a link to the Creative Commons license, and indicate if changes were made.

\section{References}

Akkina RK, Chambers TM, Nayak DP (1984) Mechanism of interference by defective-interfering particles of influenza virus: differential reduction of intracellular synthesis of specific polymerase proteins. Virus Res 1(8):687-702. https://doi.org/10.1016/0168-1702(84) 90059-5

Brooke CB, Ince WL, Wrammert J, Ahmed R, Wilson PC, Bennink JR, Yewdell JW (2013) Most influenza a virions fail to express at least one essential viral protein. J Virol 87(6):3155-3162. https://doi.org/ 10.1128/JVI.02284-12

Chung EH (2013) Vaccine allergies. Clin Exp Vaccine Res 3(1):50-57. https://doi.org/10.7774/cevr.2014.3.1.50.

Crumpton WM, Avery RJ, Dimmock NJ (1981) Influence of the host cell on the genomic and subgenomic RNA content of defectiveinterfering influenza virus. J Gen Virol 53(Pt 1):173-177. https:/ doi.org/10.1099/0022-1317-53-1-173

De BK, Nayak DP (1980) Defective interfering influenza viruses and host cells: establishment and maintenance of persistent influenza virus infection in MDBK and HeLa cells. J Virol 36(3):847-859

Dimmock NJ, Easton AJ (2015) Cloned defective interfering influenza RNA and a possible pan-specific treatment of respiratory virus diseases. Viruses 7(7):3768-3788. https://doi.org/10.3390/v7072796

Dimmock NJ, Rainsford EW, Scott PD, Marriott AC (2008) Influenza virus protecting RNA: an effective prophylactic and therapeutic antiviral. J Virol 82(17):8570-8578. https://doi.org/10.1128/JVI. 00743-08

Dimmock NJ, Dove BK, Scott PD, Meng B, Taylor I, Cheung L, Hallis B, Marriott AC, Carroll MW, Easton AJ (2012) Cloned defective interfering influenza virus protects ferrets from pandemic 2009 influenza A virus and allows protective immunity to be established. PLoS One 7(12):e49394. https://doi.org/10.1371/journal.pone. 0049394

Easton AJ, Scott PD, Edworthy NL, Meng B, Marriott AC, Dimmock NJ (2011) A novel broad-spectrum treatment for respiratory virus infections: influenza-based defective interfering virus provides protection against pneumovirus infection in vivo. Vaccine 29(15):2777-2784. https://doi.org/10.1016/j.vaccine.2011.01.102

Frensing T, Pflugmacher A, Bachmann M, Peschel B, Reichl U (2014) Impact of defective interfering particles on virus replication and antiviral host response in cell culture-based influenza vaccine production. Appl Microbiol Biotechnol 98(21):8999-9008. https:// doi.org/10.1007/s00253-014-5933-y

Genzel Y, Reichl U (2007) Vaccine production-state of the art and future needs in upstream processing. In: Pörtner R (ed) Methods in biotechnology: animal cell biotechnologymethods and protocols, vol 24, 2nd. Humana Press Inc., Totowa, pp 457-473

Genzel Y, Reichl U (2009) Continuous cell lines as a production system for influenza vaccines. Expert Rev Vaccines 8(12):1681-1692. https://doi.org/10.1586/erv.09.128

Genzel Y, Rödig J, Rapp E, Reichl U (2014) Vaccine production: upstream processing with adherent or suspension cell lines. Methods Mol Biol 1104:371-393. https://doi.org/10.1007/978-1-62703-733423

Gould PS, Easton AJ, Dimmock NJ (2017) Live attenuated influenza vaccine contains substantial and unexpected amounts of defective viral genomic RNA. Viruses 21;9(10). doi: https://doi.org/10.3390/ v9100269.

Hegde NR (2015) Cell culture-based influenza vaccines: a necessary and indispensable investment for the future. Hum Vaccin Immunother 11(5):1223-1234. https://doi.org/10.1080/21645515.2015.1016666

Heldt FS, Frensing T, Pflugmacher A, Gröpler R, Peschel B, Reichl U (2013) Multiscale modeling of influenza A virus infection supports the development of direct-acting antivirals. PLoS Comput Biol 9(11):e1003372. https://doi.org/10.1371/journal.pcbi.1003372

Jordan I, Vos A, Beilfuss S, Neubert A, Breul S, Sandig V (2009) An avian cell line designed for production of highly attenuated viruses. Vaccine 27(5):748-756. https://doi.org/10.1016/j.vaccine.2008.11. 066

Kalbfuss B, Knöchlein A, Kröber T, Reichl U (2008) Monitoring influenza virus content in vaccine production: precise assays for the quantitation of hemagglutination and neuraminidase activity. Biologicals 36(3):145-161. https://doi.org/10.1016/j.biologicals. 2007.10.002

Lohr V, Rath A, Genzel Y, Jordan I, Sandig V, Reichl U (2009) New avian suspension cell lines provide production of influenza virus and MVA in serum-free media: studies on growth, metabolism and virus propagation. Vaccine 27(36):4975-4982. https://doi.org/10.1016/j. vaccine.2009.05.083

Lohr V, Genzel Y, Jordan I, Katinger D, Mahr S, Sandig V, Reichl U (2012) Live attenuated influenza viruses produced in a suspension process with avian AGE1.CR.pIX cells. BMC Biotechnol 12(1):79. https://doi.org/10.1186/1472-6750-12-79

Mc Mahon A, Martin-Loeches I (2017) The pharmacological management of severe influenza infection - 'existing and emerging therapies'. Expert Rev Clin Pharmacol 10(1):81-95. https://doi.org/10. 1080/17512433.2017.1255550

Minor PD, Engelhardt OG, Wood JM, Robertson JS, Blayer S, Colegate T, Fabry L, Heldens JG, Kino Y, Kistner O, Kompier R, Makizumi K, Medema J, Mimori S, Ryan D, Schwartz R, Smith JS, Sugawara K, Trusheim H, Tsai TF, Krause R (2009) Current challenges in implementing cellderived influenza vaccines: implications for production and regulation, July 2007, NIBSC, Potters Bar, UK. Vaccine 27(22):2907-2913. https://doi.org/10.1016/j.vaccine.2009.02. 064

Nayak DP, Chambers TM, Akkina RK (1985) Defective-interfering (DI) RNAs of influenza viruses: origin, structure, expression, and interference. Curr Top Microbiol Immunol 114:103-151

Perdue ML, Arnold F, Li S, Donabedian A, Cioce V, Warf T, Huebner R (2011) The future of cell culture-based influenza vaccine production. Expert Rev Vaccines 10(8):1183-1194. https://doi.org/10. 1586/erv. 11.82

Peschel B, Frentzel S, Laske T, Genzel Y, Reichl U (2013) Comparison of influenza virus yields and apoptosis-induction in an adherent and a 
suspension MDCK cell line. Vaccine 31(48):5693-5699. https://doi. org/10.1016/j.vaccine.2013.09.051

Scott PD, Meng B, Marriott AC, Easton AJ, Dimmock NJ (2011a) Defective interfering influenza virus confers only short-lived protection against influenza virus disease: evidence for a role for adaptive immunity in DI virus-mediated protection in vivo. Vaccine 29(38): 6584-6591. https://doi.org/10.1016/j.vaccine.2011.06.114

Scott PD, Meng B, Marriott AC, Easton AJ, Dimmock NJ (2011b) Defective interfering influenza A virus protects in vivo against disease caused by a heterologous influenza B virus. J Gen Virol 92(Pt 9):2122-2132. https://doi.org/10.1099/vir.0.034132-0.
Stauffer Thompson KA, Rempala GA, Yin J (2009) Multiple-hit inhibition of infection by defective interfering particles. J Gen Virol 90(Pt 4):888-899. https://doi.org/10.1099/vir.0.005249-0.

WHO (1995) Cell culture as a substrate for the production of influenza vaccines: memorandum from a WHO meeting. Bull OMS 73(4): 431-435

World Health Organization (2017). Fact sheet on influenza from November 2016. http://www.who.int/mediacentre/factsheets/fs211/ en/. Accessed 23 May 2017 